\title{
Variation in Metabolic Rate among Individuals Is Related to Tissue-Specific Differences in Mitochondrial Leak Respiration
}

\author{
Karine Salin ${ }^{1, *}$ \\ Sonya K. Auer ${ }^{1}$ \\ Agata M. Rudolf ${ }^{2}$ \\ Graeme J. Anderson ${ }^{1}$ \\ Colin Selman ${ }^{1}$ \\ Neil B. Metcalfe ${ }^{1}$ \\ ${ }^{1}$ Institute of Biodiversity, Animal Health, and Comparative \\ Medicine, College of Medical, Veterinary, and Life Sciences, \\ University of Glasgow, Glasgow G12 8QQ, United Kingdom; \\ ${ }^{2}$ Institute of Environmental Sciences, Jagiellonian University, \\ Gronostajowa 7, 30-387 Krakow, Poland
}

Accepted 8/7/2016; Electronically Published 9/27/2016

\begin{abstract}
Standard metabolic rate (SMR) and maximum metabolic rate (MMR) typically vary two- or threefold among conspecifics, with both traits assumed to significantly impact fitness. However, the underlying mechanisms that determine such intraspecific variation are not well understood. We examined the influence of mitochondrial properties on intraspecific variation in SMR and MMR and hypothesized that if SMR supports the cost of maintaining the metabolic machinery required for MMR, then the mitochondrial properties underlying these traits should be shared. Mitochondrial respiratory capacity (leak and phosphorylating respiration) and mitochondrial content (cytochrome $c$ oxidase activity) were determined in the liver and white muscle of brown trout Salmo trutta of similar age and maintenance conditions. SMR and MMR were uncorrelated across individuals and were not associated with the same mitochondrial properties, suggesting that they are under the control of separate physiological processes. Moreover, tissue-specific relationships between mitochondrial properties and whole-organism metabolic traits were observed. Specifically, SMR was positively associated with leak respiration in liver mitochondria, while MMR was positively associated with muscle mitochondrial leak respiration and mitochondrial content. These results suggest that a high SMR or MMR, rather than signaling a higher ability for respiration-driven ATP synthesis, may actually reflect greater dissipation of energy, driven by proton leak across the mitochondrial inner membrane. Knowledge of these links should aid interpretation of the potential fitness conse-
\end{abstract}

${ }^{*}$ Corresponding author; e-mail: salin.karine@gmail.com.

Physiological and Biochemical Zoology 89(6):000-000. 2016. (C) 2016 by The University of Chicago. All rights reserved. 1522-2152/2016/8906-5122\$15.00. DOI: $10.1086 / 688769$ quences of such variation in metabolism, given the importance of mitochondria in the utilization of resources and their allocation to performance.

Keywords: brown trout, fish, liver, oxygen consumption, white muscle.

\section{Introduction}

Aerobic metabolism is a key feature of an organism's physiology: its role in governing resource flux means that it determines the rate at which resources can be consumed and allocated to performance-related traits (Hulbert and Else 2000; Brown et al. 2004; Mathot and Dingemanse 2015). Two fundamental physiological parameters define the floor and ceiling of aerobic metabolic capacity. The lowest levels of metabolic activity are called standard and basal metabolic rates (SMR and BMR, respectively) and are defined as the oxygen consumption of a resting, postabsorptive, and nonreproductive organism at a given temperature for an ectotherm and at thermoneutrality for an endotherm, respectively (Willmer et al. 2005). At the other end of the metabolic continuum, maximum metabolic rate (MMR) is the maximal short-term aerobic capacity of an individual animal (Willmer et al. 2005). The difference between SMR or BMR and MMR provides a measure of the animal's range of aerobic capacity above its baseline energetic needs, termed the aerobic scope (AS; Fry 1947).

The concept of AS provides insight into an animal's metabolic limitations since it determines the extent to which it can allocate energy to growth and reproduction (Guderley and Pörtner 2010). Therefore, AS may have a significant impact on fitness but displays significant intraspecific variation (Farrell et al. 2008; Sandblom et al. 2014; Auer et al. 2015a, 2015c; but see Norin et al.2014). Both minimum and maximum metabolic rate are responsible for intraspecific variation in AS since they exhibit consistent differences among individuals over extended periods of time, even within a population under standardized conditions (Gomes et al. 2004; Burton et al. 2011; Swanson et al. 2012; Careau et al. 2014a, 2014b; Gifford et al. 2014). In turn, the use of a single metric such as AS conceals a great deal of variation, since individuals with the same AS can have markedly different absolute levels of minimum and maximum metabolic rate (Auer et al. 2015c). Because natural selection works on AS variation between individuals within a population, it is of special interest to elucidate the underlying mechanisms behind variation in minimum and maximal metabolic rates. 
Minimum and maximum metabolic rates are predicted to be positively correlated with one another since SMR or BMR is thought to reflect the maintenance costs of the metabolic machinery needed to support MMR capacity (Bennett and Ruben 1979; Biro and Stamps 2010). However, while a positive correlation between minimum and maximum metabolic rates at the intraspecific level has indeed been found in some contexts (Gomes et al. 2004; Biro and Stamps 2010; Careau et al. 2014a, $2014 b$ ), this is not the case in others (Chappell et al. 2004, 2007; Gomes et al. 2004; Careau et al. 2014b). The mechanisms underlying this purported link are also equivocal, with some studies reporting a positive relationship between metabolic rates and underlying traits such as organ size (Konarzewski and Ksiazek 2013) or mitochondrial density (Norin and Malte 2012), whereas others find no relationship (Selman et al. 2001; Chappell et al. 2007; Larsen et al. 2011; Norin and Malte 2012; Boldsen et al. 2013). This makes it impossible to explain mechanisms behind variation in AS, since it could be a consequence of variation in minimum and maximum metabolic rates that is either concomitant and connected or independent and separated. This lack of consistency suggests that there is a need to look at processes on a more fundamental level to understand what determines variation in SMR and MMR among conspecific living under the same conditions.

Mitochondria are responsible for almost $90 \%$ of cellular respiration (Rolfe and Brand 1996), so variation in their respiratory capacities may help explain individual variation in SMR and MMR. Mitochondria must continually consume oxygen in order to maintain the proton gradient across the inner mitochondrial membrane, which is continually being dissipated by two major processes: proton leak across the inner mitochondrial membrane and the passage of protons through the ATP synthase that drives ATP production through phosphorylation of ADP (Brand and Nicholls 2011). These two processes are measured ex vivo as the leak respiration rate (LEAK; also described as state 4) and phosphorylating respiration rate (OXPHOS; state 3 ), respectively (Chance and Williams 1955).

While conditions for LEAK (unlimited availability of energy substrates in the absence of ATP synthesis) and OXPHOS (unlimited availability of energy substrates, oxygen, and ADP) do not strictly correspond to those found in mitochondria in vivo, LEAK and OXPHOS clearly influence minimum and maximum rates of whole-body metabolism. Intraspecific variability in LEAK and OXPHOS can be considerable and is positively related to SMR and BMR (Brown et al. 2012; Salin et al. 2012; Monternier et al. 2014) and MMR (Coen et al. 2012; Jacobs et al. 2012; Schlagowski et al. 2013). These investigations into the effects of these mitochondrial properties on minimum or maximum metabolic rate have generally focused on comparisons of mean values between treatment groups or life stages (Brown et al. 2012; Coen et al. 2012; Jacobs et al. 2012; Khan et al. 2014; Monternier et al. 2014). However, accounting for individual heterogeneity within a population is critical for understanding properties affecting fitness, since individual variation is what natural selection acts on (Careau and Garland 2012; Vindenes and Langangen 2015). Studies are therefore needed to investigate the mitochondrial processes underlying variation in MMR and SMR or BMR (and their covariation) among conspecifics living under the same conditions.

Previous studies examining the relationship between different mitochondrial properties and rates of whole-body metabolism have typically focused on a single tissue (Brookes et al. 1998; Chung et al. 2011; Larsen et al. 2011; Coen et al. 2012; Jacobs et al. 2012; Schlagowski et al. 2013; but see Norin and Malte 2012). However, the link between mitochondrial properties and metabolic traits may be tissue specific for two main reasons (Amara et al. 2007; Trzcionka et al. 2008; Brown et al. 2012). First, mitochondrial respiratory capacities differ significantly among tissues (Hulbert et al. 2006; Park et al. 2014). Second, the relative contribution of a tissue to whole-organism oxygen consumption may change as a function of the metabolic state of the organism. At the onset of physical exercise inducing MMR, there is a redistribution of blood flow from inactive tissues (e.g., liver) to active tissues (e.g., muscle; Willmer et al. 2005). This shift can generate different tissue-specific contributions of mitochondrial properties to MMR versus SMR or BMR. Thus, an understanding of the links between mitochondrial properties, minimum metabolic rate, and MMR requires examination of intraspecific variation in mitochondrial processes in more than a single tissue, but this has never previously been attempted.

We examined SMR and MMR in juvenile brown trout (Salmo trutta) and determined whether individual variation in these metabolic traits could be explained by their mitochondrial properties. We tested the predictions that (i) higher SMR and MMR are explained by higher oxygen requirements of the mitochondria and (ii) if the relationship between SMR and MMR is significantly positive, then we would expect shared underlying mitochondrial properties between the two metabolic traits. Alternatively, (iii) if metabolic traits are not correlated, we predict that tissues and/or mitochondrial properties differ in how they influence SMR and MMR. We chose brown trout as our study organism because they are known to exhibit consistent differences in SMR and MMR among individuals of the same age maintained under standardized conditions (Norin and Malte 2011; Auer et al. 2015b). We analyzed mitochondrial properties in the liver and the white muscle, as these tissues account for a large proportion of an individual's overall metabolic rate by virtue of their mass-specific oxygen consumption and relative mass, respectively (Porter and Brand 1995; Coen et al. 2012).

\section{Material and Methods}

\section{Fish Origin and Care}

Juvenile brown trout (Salmo trutta) were captured from the wild and allowed to acclimate for $1 \mathrm{mo}$ in a holding tank $\left(11.5^{\circ} \mathrm{C}\right)$ under controlled conditions. In order to standardize their nutritional state, fish were transferred to individual compartments where they were fed twice daily on individual rations for the next $9 \mathrm{wk}$, except for the $48 \mathrm{~h}$ preceding both the metabolic rates and the 
mitochondrial measurement, when no food was provided as described for these same individuals in Auer et al. (2015c). Rations were calculated based on body size and derived from equations that permitted growth but at a submaximal rate (Elliott 1976). Body size was measured every $2 \mathrm{wk}$, and rations were recalculated to adjust for growth (see appendix for full details of fish origin and care and the calculation of their food rations).

\section{Whole-Organism Oxygen Consumption}

After 8 wk on these standardized rations, we measured their SMR and MMR using protocols described in Auer et al. (2015c). Briefly, SMR was measured using continuous flow-through respirometry techniques whereby oxygen consumption rates of fish within individual chambers were measured over a 20 -h period. We then determined the MMR of each fish by measuring its oxygen consumption in a closed respirometry system immediately following exhaustive exercise. The exhaustion protocol consisted of continuously chasing the fish against a circular current (Norin and Malte 2012), with fish being defined as exhausted when they could no longer swim, were unresponsive, and did not resist being picked up by hand. Both metabolic measurements were found to be repeatable when the same group of fish as used in this experiment were maintained on a stable food regime (repeatability of SMR: $r=0.78, n=38, P<0.001$; MMR: $r=0.45$, $n=38, P=0.039$; Auer et al. 2015c). SMR and MMR are expressed in milligrams of oxygen per hour. (See appendix for full details of SMR and MMR measurement and data analysis.)

\section{Mitochondrial Properties in White Muscle and Liver}

At week 9, the fish $(12.78 \pm 0.30 \mathrm{~g}[$ mean $\pm 1 \mathrm{SE}], n=38)$ were culled, and subsamples of their white skeletal muscle and liver were immediately collected, weighed, and homogenized in icecold MiR05 respirometry buffer (see appendix for the buffer composition) for analyses of mitochondrial properties. Total liver mass was also recorded. The technique for preparation of the white muscle and liver homogenate was adapted from Kuznetsov et al. (2002, 2008; Larsen et al. 2014) and is detailed in the appendix and in Salin et al. (2016). The homogenates were diluted further in MiR05 to obtain the desired final concentration $\left(10 \mathrm{mg} \mathrm{mL}^{-1}\right.$ for the liver and $20 \mathrm{mg} \mathrm{mL}^{-1}$ for the white muscle). Two replicate samples of liver or muscle homogenate were then added to chambers of Oxygraph-2k high-resolution respirometers (Oroboros Instruments, Innsbruck, Austria) at $12^{\circ} \mathrm{C}$. A sequential substrate/inhibitor addition protocol, adapted from Pesta and Gnaiger (2012) was run simultaneously for each individual and tissue in duplicate. Briefly, the rate of OXPHOS was assessed by adding nonlimiting ADP to the chamber containing both pyruvate and malate (complex I substrates) and succinate (complex II substrate). LEAK was then measured by inhibiting ATP synthase through the addition of oligomycin. Finally, cytochrome c oxidase (COX) activity per mass of tissue was measured in the presence of complex I inhibitor (rotenone) and complex III inhibitor (antimycin A) and after addition of ascorbate and $\mathrm{N}, \mathrm{N}, \mathrm{N}^{\prime}, \mathrm{N}^{\prime}$-tetramethyl-p-phenylenediamine dihy- drochloride (see appendix for full details of the titration protocol, oxygraph calibration, oxygen flux, and substrate concentrations). COX, an inner mitochondrial membrane enzyme involved in the electron transport chain, is a marker of mitochondrial content and is highly correlated to mitochondrial respiratory capacity (Larsen et al. 2012). Oxygen consumption at each step in the protocol was averaged over several minutes after stabilization of the flux, and the mass-specific mitochondrial respiration rate was expressed in picomoles of oxygen per second per milligram wet weight of tissue (Pesta and Gnaiger 2012).

\section{Data Analysis}

We first calculated intraclass correlation coefficients to determine whether mitochondrial respiration measures were repeatable in the two replicate tissue samples taken for liver and muscle. Pearson's $r$ was used to examine whether mass-specific mitochondrial properties (LEAK per mg of tissue, OXPHOS per mg of tissue, and COX activity per mg of tissue) were correlated between the liver and white muscle of the same fish. At the level of the whole animal, we then used regression analysis to determine the relationships between double $\log _{10}$-transformed body mass and SMR or MMR (table A1). Residuals from these regressions were used as mass-independent estimates of wholeorganism oxygen consumption (rSMR and rMMR) in correlation analyses to test whether rSMR and rMMR were correlated within a fish.

Second, we examined whether variation among fish in SMR and then MMR was correlated with the properties of the mitochondria in the liver and/or muscle. Specifically, we tested whether the respiratory capacities of the mitochondria and/or the mitochondrial content in those two tissues explained individual variation in whole-organism oxygen consumption. To avoid a collinearity issue between the mitochondrial properties, LEAK and OXPHOS were first normalized for mitochondrial content by using residuals from the regressions relating LEAK and OXPHOS to COX activity for both liver and muscle (table A1). We used residuals from the regression instead of the fraction of mass-specific mitochondrial respiration rates per COX activity since mass-specific mitochondrial respiration rates were not isometrically related to COX activity. These normalized values $\left(\mathrm{LEAK}_{\mathrm{COX}}\right.$ and OXPHOS $\mathrm{COX}_{\mathrm{C}}$ ) were then used as respiratory capacities of the mitochondria in subsequent analyses. Such an approach allowed us to simultaneously consider all the mitochondrial properties (variation in LEAK, OXPHOS, and COX activity) as predictors of whole-animal oxygen consumption within a single model rather than run multiple separate analyses for each mitochondrial property. We used separate linear regressions to determine the dependencies of first log-transformed SMR and then log-transformed MMR on log-transformed body mass, respiratory capacities, and content of the mitochondria $\left(\mathrm{LEAK}_{\mathrm{COX}}, \mathrm{OXPHOS}_{\mathrm{COX}}\right.$, and COX activity for both liver and white muscle).

In separate models, we tested whether variation among fish in SMR and MMR was correlated with the mitochondrial properties 
of the entire liver. Specifically, we multiplied the mass-specific mitochondrial properties by the total liver mass (LEAK per liver, OXPHOS per liver, and COX activity per liver). As above, the LEAK and OXPHOS of the entire liver were normalized for mitochondrial content of the liver by using residuals from the regressions relating LEAK and OXPHOX of total liver to COX activity of total liver (table A1). We used separate linear regressions to determine the dependencies of first log-transformed SMR and then log-transformed MMR on log-transformed body mass and mitochondrial properties of the entire liver. The results were qualitatively the same as those obtained when mitochondrial capacities of the liver were expressed in mass-specific terms, so only those for mass-specific mitochondrial properties are reported in the text (see table A3 for analysis based on total liver properties).

Output from the linear regression models is presented as unstandardized coefficients $\pm 1 \mathrm{SE}$ without removing nonsignificant terms. Partial correlations were calculated and squared for each significant predictor variable. All analyses are based on a sample size of 38 fish, with the exception of those analyses including COX respiration rate and COX-normalized respiration in the liver, where the sample size was 37 because COX activity was not measured in one individual. Variance inflation factors for all predictors of each regressions analysis were less than 2. All statistical analyses were performed in IBM SPSS Statistics 21 (SPSS, Chicago); the level of significance was set to $P<0.05$, and all values are presented as mean $\pm 1 \mathrm{SE}$.

\section{Results}

Mass-specific leak and phosphorylating respiration rates and mass-specific COX activity were highly repeatable across replicate samples within each tissue but varied two- or threefold across individuals for each tissue (table A2). All mass-specific mitochondrial respiration properties of the liver were approximately double those of the white muscle (table A2). However, the massspecific properties of the liver and muscle mitochondria from the same fish were not correlated (table 1). SMR and MMR increased with body mass, as expected, but differed up to twofold among individuals of the same body mass (fig. 1). After controlling for body mass, there was no correlation between SMR and MMR (Pearson's $r=0.035, P=0.833$ ).

Table 1: Correlation coefficients of mass-specific mitochondrial properties (leak respiration rate [LEAK], phosphorylating respiration rate [OXPHOS], and cytochrome c oxidase [COX] activity) for the liver versus the equivalent mitochondrial property in white skeletal muscle from the same fish

\begin{tabular}{lcc}
\hline & Pearson's $r$ & $P$ \\
\hline LEAK & .059 & .730 \\
OXPHOS & .159 & .347 \\
COX activity & .219 & .192 \\
\hline
\end{tabular}

Note. $n=38$ fish for respiration rates, 37 fish for COX activity. Oxygen fluxes were measured as picomoles of oxygen per second per milligram of wet mass at $12^{\circ} \mathrm{C}$ at a final homogenate concentration of $10 \mathrm{mg} \mathrm{mL}^{-1}$ for the liver and $20 \mathrm{mg} \mathrm{mL}^{-1}$ for the skeletal muscle.

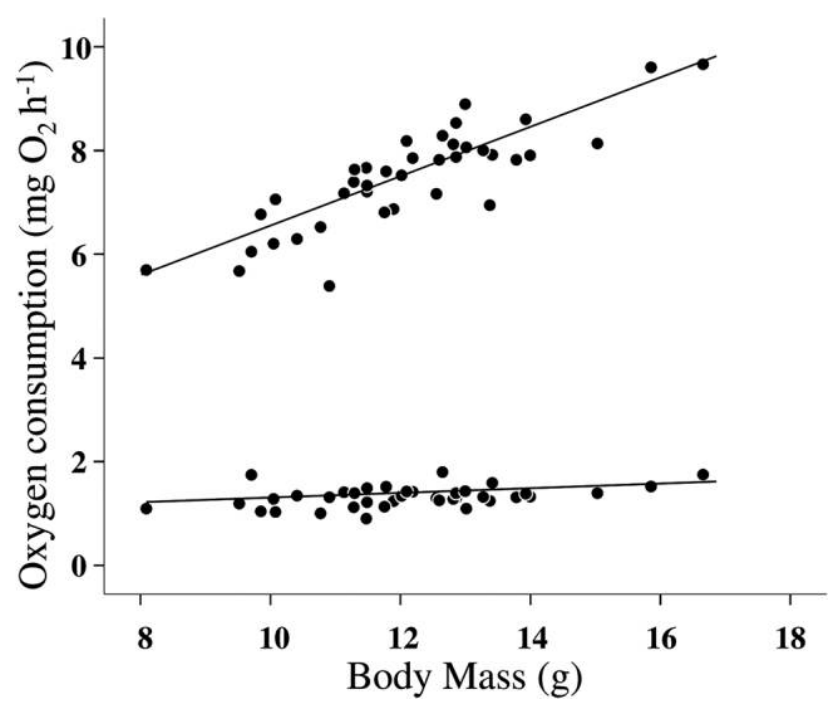

Figure 1. Relationships between standard metabolic rate (SMR), maximum metabolic rate (MMR), and body mass of brown trout Salmo trutta. Residuals (rSMR and rMMR) generated from these regressions were used to distinguish individuals with an above-average metabolic rate for their body mass, that is, those with positive residuals, from those with below-average metabolic rates for their body mass, that is, negative residual values.

An individual's SMR was explained only by its liver LEAK $_{\text {Cox }}\left(r^{2}=0.135\right.$; table 2; fig. 2$)$ once the effect of body mass was taken into account. SMR was not related to $\mathrm{LEAK}_{\mathrm{COX}}$ in the muscle or $\mathrm{OXPHOS}_{\mathrm{COX}}$ in either the liver or the muscle (table 2). Additionally, there was no evidence that SMR was linked to the COX activity (i.e., amount of mitochondria) in either tissue (table 2). In contrast, MMR was found to be unrelated to any liver mitochondrial properties (table 2) but was positively associated with both $\mathrm{LEAK}_{\mathrm{COx}}$ and COX activity of the white muscle ( $r^{2}=0.162$ and $r^{2}=0.135$, respectively; fig. $3 A, 3 B$; table 2 ) once the effect of body mass was taken into account.

\section{Discussion}

In this study we observed two- or threefold differences among individual fish in their SMR and MMR, with a similar scale of variation observed in mitochondrial properties. This variation occurred despite the experimental animals' being of similar age, nutritional state, and body size and having been maintained under identical environmental conditions. We found no correlation between SMR and MMR among fish or between the mitochondrial properties of the liver and white muscle. The absence of covariation between tissues supports earlier findings (Garland 1984; Garland and Else 1987; Park et al. 2014), while the lack of any correlation between minimum metabolic rate and MMR has also been found elsewhere (Chappell et al. 2004, 2007; Gomes et al. 2004; Careau et al. 2014b). However, a highly original finding was the evidence of clear differences between liver and muscle in terms of the relationship between their mitochondrial properties and whole-organism metabolic 
Table 2: Results from linear regression analyses of log-transformed standard metabolic rate (log SMR) and maximum metabolic rate (rMMR) as a function of log-transformed body mass and mitochondrial properties (cytochrome c oxidase [COX] activity, COX-normalized leak respiration rate $\left[\mathrm{LEAK}_{\mathrm{COX}}\right]$, COX-normalized phosphorylating respiration rate $\left.\left[\mathrm{OXPHOS}_{\mathrm{COX}}\right]\right)$ in brown trout (Salmo trutta)

\begin{tabular}{|c|c|c|c|c|c|c|c|c|}
\hline \multirow[b]{2}{*}{ Predictors } & \multicolumn{4}{|c|}{ Log SMR } & \multicolumn{4}{|c|}{ Log MMR } \\
\hline & $\begin{array}{c}\text { Parameter estimate } \\
\pm 1 \mathrm{SE}\end{array}$ & $t$ & df & $P$ & $\begin{array}{c}\text { Parameter estimate } \\
\pm 1 \mathrm{SE}\end{array}$ & $t$ & df & $P$ \\
\hline Log body mass & $.508 \pm .176$ & 2.888 & 29 & .007 & $.838 \pm .089$ & 9.417 & 29 & $<.001$ \\
\hline Liver LEAK $_{\mathrm{COx}}$ & $.049 \pm .023$ & 2.133 & 29 & .042 & $-.010 \pm .012$ & -.852 & 29 & .401 \\
\hline Liver OXPHOS & $.001 \pm .005$ & .246 & 29 & .807 & $.002 \pm .003$ & .898 & 29 & .377 \\
\hline Liver COX activity & $-.001 \pm .002$ & -.790 & 29 & .436 & $.000 \pm .001$ & .079 & 29 & .938 \\
\hline Muscle LEAK $_{\mathrm{COx}}$ & $-.003 \pm .050$ & -.052 & 29 & .959 & $.060 \pm .025$ & 2.372 & 29 & .025 \\
\hline Muscle OXPHOS & $-.003 \pm .050$ & -.192 & 29 & .849 & $-.009 \pm .007$ & -1.318 & 29 & .198 \\
\hline Muscle COX activity & $.003 \pm .003$ & .955 & 29 & .347 & $.004 \pm .002$ & 2.127 & 29 & .042 \\
\hline
\end{tabular}

Note. Mitochondrial oxygen fluxes were measured as picomoles of oxygen per second per milligram of wet mass at $12^{\circ} \mathrm{C}$ at a final homogenate concentration of $10 \mathrm{mg} \mathrm{mL}^{-1}$ for the liver and $20 \mathrm{mg} \mathrm{mL}^{-1}$ for the skeletal muscle. Bold denotes significance.

rates. This suggests that the hypothesized link between SMR and MMR (i.e., that a significant component of minimum metabolic rate is the cost of maintaining the machinery needed to sustain MMR; Bennett and Ruben 1979; Biro and Stamps 2010) is perhaps too simplistic when examining variability among individuals kept under the same conditions. The processes driving the two components of AS thus appear distinct.

The significant intraspecific relationship between MMR and the mitochondrial properties of the white muscle is not unexpected. This is because MMR was measured during peak excess postexercise oxygen consumption (EPOC), when the major requirement for oxygen is in the removal of lactate and the restoration of cellular homeostasis, which is supported by white muscle (Wood 1991). However, given that white muscle contributes approximately $35 \%$ to the total body mass of a trout (calculated according to Houlihan et al. 1986), it is perhaps surprising that white muscle mitochondria did not explain any of the substantial variation in SMR, in contrast to that relationship reported in other species (Rolfe and Brand 1996; Monternier et al. 2014). Instead, only the bioenergetics of the liver contributed to the variability in SMR. Additional support for such a link is found in mammalian species, where the liver also contributes to a disproportionately large fraction of basal metabolic rate (Rolfe and Brown 1997). Our observations illustrate that an understanding of interindividual variability in SMR and MMR can be gained only through consideration of the mitochondrial properties of tissue specifically linked to particular whole-organism metabolic traits (John-Alder 1983; Garland 1984; Garland and Else 1987; Norin and Malte 2012).

Our study shows that individual variation in SMR and MMR is explained by corresponding variation in respiration supporting the offset of proton leakage in the mitochondria. Leak respiration in the liver was significantly greater in high-SMR individuals, while MMR was positively related to leak respiration in the muscle. These results are consistent with other studies comparing experimental groups or taxa that have shown animals with higher metabolism to have higher leak respiration (Rolfe and Brand 1997; Brookes et al. 1998; Jacobs et al. 2012; Salin et al. 2012; Monternier et al. 2014; but see Larsen et al. 2011). Though there is a need for some mitochondrial ATP to support diverse cellular activities in vivo, the low respiration rate under basal conditions (i.e., SMR or BMR) results from both basal ATP demand and the effects of mitochondrial proton leak (Kadenbach 2003; Brand and Nicholls 2011). In fact, the latter was found to be responsible for a significant proportion $(18 \%-22 \%)$ of the total oxygen consumption at rest in rats

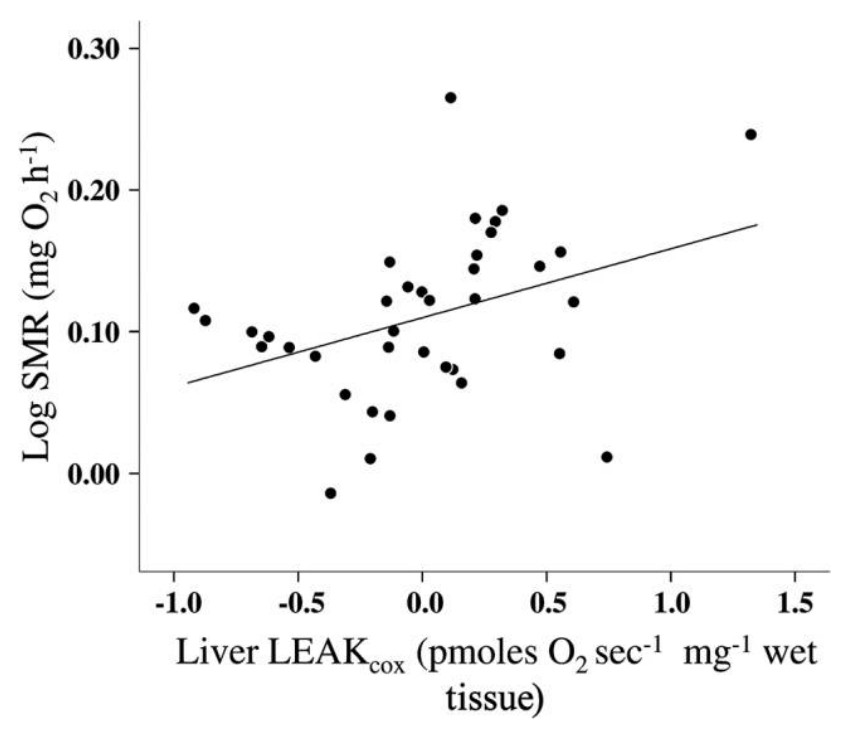

Figure 2. Relationship between log-transformed standard metabolic rate (log SMR) of brown trout (Salmo trutta) and their liver mitochondrial leak respiration rate, normalized for cytochrome c oxidase $(\mathrm{COX})$ activity $\left(\mathrm{LEAK}_{\mathrm{Cox}} ; r^{2}=0.135, P=0.042\right)$. Plotted on the $Y$ axis are partial residuals after accounting for variation in all other predictors of the linear regression analysis (see table 2 for details) and standardized for the mean fish size of $12.1 \mathrm{~g}$. 


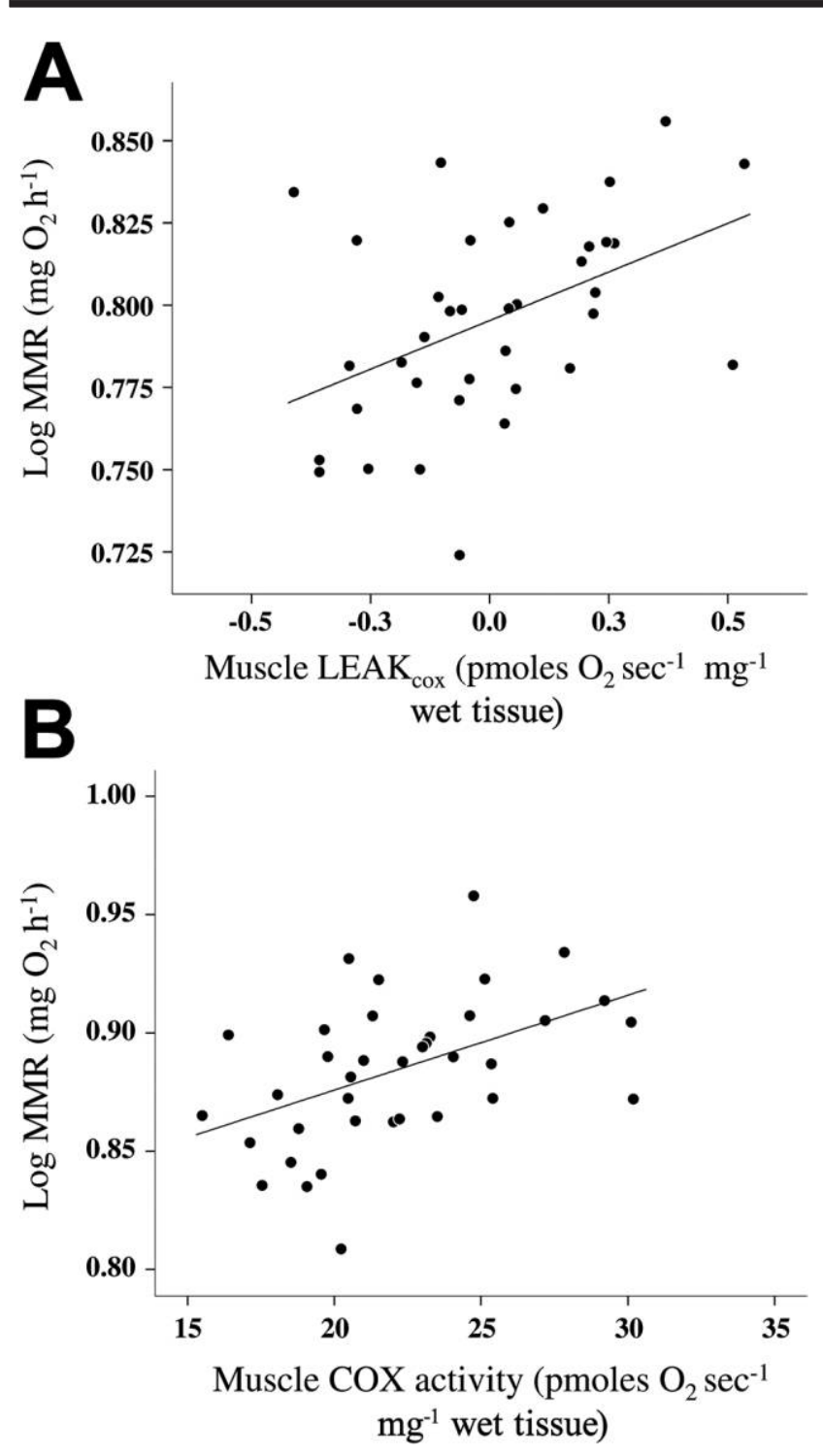

Figure 3. Relationships between log-transformed maximum metabolic rate (log MMR) of brown trout (Salmo trutta) and their white muscle mitochondrial leak respiration rate, normalized for cytochrome $\mathrm{c}$ oxidase $(\mathrm{COX})$ activity $\left(\mathrm{LEAK}_{\mathrm{Cox}} ; A ; r^{2}=0.162, P=0.025\right)$ and the mass-specific COX activity of their white muscle $\left(B ; r^{2}=0.135, P=\right.$ $0.042)$. Plotted on the $Y$-axis are partial residuals after accounting for variation in all other predictors of the linear regression analysis (see table 2 for details) and standardized for the mean fish size of $12.1 \mathrm{~g}$.

(Rolfe and Brand 1996; Brookes et al. 1998). The major contribution of white muscle to MMR is due to its metabolic role in lactate clearance and glycogen resynthesis during EPOC (Wood 1991). However, it is noteworthy that the link between MMR and mitochondrial capacities in this study was with LEAK rather than OXPHOS respiration. Interestingly, the mitochondria of white muscle during EPOC have been found to respire at a rate closer to LEAK than OXPHOS (Moyes et al. 1992), which may explain why the mitochondrial leak respiration of white muscle has a significant effect on MMR.
The importance of mitochondrial leak respiration in explaining intraspecific variation in SMR and MMR suggests that the leak of protons across the mitochondrial membrane may be an important mediator of the link between metabolic capacity and animal performance. The proton leak is thought to dissipate the energy of the proton gradient with consequences at the level of the whole animal, such as a potentially faster loss of body mass under conditions of food restriction (Harper et al. 2001). Even when food is not limiting, individuals with higher leak respiration have lower power output during physical exercise (Schlagowski et al. 2013) and slower growth (Salin et al. 2012). Individuals that have higher aerobic metabolic rates - and so higher mitochondrial leak respiration - may therefore have a lower efficiency of ATP production (Salin et al. 2012; Schlagowski et al. 2013). However, they may also benefit from a lower production of reactive oxygen species (ROS; Salin et al. 2015b). Such a physiological trade-off between ATP and ROS may help explain the persistence of variation in metabolic rates among individuals; metabolic phenotypes can have differential effects on fitness (Burton et al. 2011; Salin et al. 2015a). For example, we have shown that individual brown trout with relatively higher SMR perform worse under conditions of low food availability, which may be explained by a reduced capacity to generate ATP above baseline energetic needs (Auer et al. $2015 c$ ). Interestingly, when trout were fed ad lib., those individuals displaying a relatively higher SMR grew the fastest, likely because they could maintain ATP generation while benefiting from lower rates of ROS generation (Salin et al. 2015a).

Given the growing attention paid to AS as a proxy for identifying metabolic limitations as well as predicting the physiological failure of an individual, there is a need to understand the proximate mechanism(s) operating on SMR and MMR at the individual level. This study provides evidence that differences in the determinants of AS among individuals are related to tissue-specific variation in mitochondrial properties. Thus, it is clear that AS is the comprehensive integration of two distinct physiological processes, that is, those underlying SMR and MMR. One notable finding is that individuals with higher standard or maximum metabolic rates are (counterintuitively) likely to be less efficient in using their resources since they will "waste" more oxygen and energy substrate, due to the need to offset a higher proton leak. These results emphasize the importance of investigating the respiratory capacities of mitochondria since they determine an individual's requirement for energy substrates and oxygen as well as the rate at which ATP is generated to allocate to fitness-related traits (Salin et al. 2015a).

\section{Acknowledgments}

We thank T. van Leeuwen and N. Sinclair for help with fish collection; J. Orledge for equipment organization; Alyson Lewis for technical help; S. Killen for respirometry advice; two referees for helpful comments on an earlier version of the manuscript; and G. Law, A. Kirk, and R. Phillips for maintaining the fish before the experiment. All procedures were carried out under the jurisdiction of a UK Home Office Project License (PPL 60/4292). We declare no competing interests. 
This research was supported by a European Research Council Advanced Grant (number 322784) to N.B.M.

\section{APPENDIX}

\section{Supplemental Information}

\section{Supplemental Material and Methods \\ Fish Origin and Care}

Forty wild juvenile brown trout were collected by electrofishing from the River Endrick, Scotland $\left(56^{\circ} 03^{\prime} \mathrm{N}, 4^{\circ} 19^{\prime} \mathrm{W}\right)$ and transported to the University of Glasgow in August 2013. Fish were acclimated in a 400-L holding tank, kept within a temperature-controlled room $\left(11.5^{\circ} \pm 1^{\circ} \mathrm{C}\right.$; mean \pm actual range) under a $12 \mathrm{~L}: 12 \mathrm{D}$ photoperiod, and fed daily to excess with Inicio Plus trout pellets (BioMar, Grangemouth, UK). In September 2013, the fish were transferred to individual compartments within a purpose-designed stream tank system. This system, in which adjacent compartments $(190 \mathrm{~mm} \times$ $130 \mathrm{~mm} \times 200 \mathrm{~mm}$ ) were separated by mesh screens, allowed us to feed fish individually by hand twice per day (at 0900 and 1600 hours) while maintaining them in flowing water of the same quality and temperature (Auer et al. 2015c). Fish were fed an intermediate ration of pellets using equations from Elliott (1976) that describe the growth of brown trout as a function of caloric intake, water temperature, and initial body mass (see Auer et al. $2015 c$ for more details). Specifically, the daily ration was calculated as calories $=16.3 W^{0.737} e^{(0.154 T)}$, where $W$ is body mass $(\mathrm{g})$ and $T$ is water temperature.

\section{Experimental Protocol}

Fish were briefly anesthetized $\left(50 \mathrm{mg} \mathrm{L}^{-1}\right.$ benzocaine diluted in water) for measurement of body mass every $2 \mathrm{wk}$ (at days 0 , $14,28,42$, and 56) using a 0.001 -g precision scale (Sartorius plus balance) to allow adjustment of food rations as the fish grew. Standard and maximum metabolic rates (SMR and MMR, respectively) were measured on day 56 of the experiment (after $2 \mathrm{~d}$ of food deprivation). Fish were then allowed $7 \mathrm{~d}$ of recovery after the MR measurement before being culled for mitochondrial assessment; this duration was shown to be sufficient in salmonids for a total elimination of the anesthetic (Allen 1988) used when weighing fish after respirometry and recovery of the endocrine and energy parameters from the stress of handling (Pickering et al. 1982).

The liver (excluding the gall bladder) and a sample of skeletal white muscle, collected between the dorsal and caudal fins, were immediately dissected. A subsample of each tissue was cleaned of fat and connective tissue, weighed (0.001-g precision; Explorer Ohaus balance), and transferred to icecold MiR05 respirometry buffer $(0.5 \mathrm{mM}$ EGTA, $3.0 \mathrm{mM}$ $\mathrm{MgCl}_{2} 6 \mathrm{H}_{2} \mathrm{O}, 20 \mathrm{mM}$ taurine, $10 \mathrm{mM} \mathrm{KH}_{2} \mathrm{PO}_{4}, 20 \mathrm{mM}$ HEPES, $110 \mathrm{mM}$ D-sucrose, $60 \mathrm{mM}$ lactobionic acid, $1 \mathrm{~g} \mathrm{~L}^{-1}$ bovin serum albumin essentially free fatty acid, $\mathrm{pH}$ 7.3) to determine mitochondrial function (see below).
Whole-Organism Respirometry

SMR and MMR were measured as the rate of whole-body oxygen consumption at $11.5^{\circ} \pm 0.5^{\circ} \mathrm{C}$ (mean \pm actual range) following a 48-h fasting period to exclude the effects of specific dynamic action (Rosenfeld et al. 2015). SMR was measured first, in undisturbed fish in an open-flow system over a 20 -h period. Full details are given in Auer et al. (2015c), but, in brief, fish were kept in individual respirometry chambers in the dark, with an empty chamber being used as a control. Air-saturated water was pulled from the chambers with a peristaltic pump at $1.68 \mathrm{~L} \mathrm{~h}^{-1}$ to a fiberoptic oxygen sensor (robust probe; PyroScience, Aachen, Germany). Oxygen, temperature, and barometric pressure data were read by multichannel oxygen meters (FireStingO2, PyroScience) and recorded using FireSting software, version 3.0 (PyroScience).

Oxygen consumption rate $\left(\mathrm{mg} \mathrm{O}_{2} \mathrm{~h}^{-1}\right)$ was calculated according to the equation

$$
\dot{\mathrm{M}}_{2}=V_{\mathrm{w}} \times\left(C_{\mathrm{w} \mathrm{O}_{2} \text { control }}-C_{\mathrm{w} \mathrm{O}_{2} \text { fish }}\right)
$$

where $V_{\mathrm{w}}$ is the flow rate of water through the respirometry chamber $\left(\mathrm{L} \mathrm{h}^{-1}\right)$ and $C_{\mathrm{wO}_{2}}$ control and $C_{\mathrm{w}} \mathrm{O}_{2}$ fish are the concentrations of oxygen $\left(\mathrm{mg} \mathrm{L}^{-1}\right)$ in the outflow of the chambers lacking and containing fish, respectively, after adjusting for temperature and barometric pressure. SMR for each fish was calculated by taking the mean of the lowest tenth percentile of oxygen consumption measurements over the 20 -h measurement period and then excluding outliers, that is, those measurements below $2 \mathrm{SD}$ from this mean (Clark et al. 2013).

Following the SMR measurement, MMR was measured in fish recovering from exhaustive exercise (Norin and Malte 2012). They were then immediately transferred into a respirometry chamber connected to a closed and recirculating system. The oxygen concentration was measured over a 5-min test period using the same equipment and temperature as for the SMR explained above. Oxygen consumption rate was calculated as

$$
\dot{\mathrm{Mo}}_{2}=\left(V_{\mathrm{r}}-V_{\mathrm{f}}\right) \times \frac{\Delta C_{\mathrm{w}} \mathrm{O}_{2}}{\Delta t},
$$

where $V_{\mathrm{r}}$ is the volume of the respirometry system and $V_{\mathrm{f}}$ is the volume of the fish, assuming $1 \mathrm{~g}$ of fish is equivalent to $1 \mathrm{~mL}$ of water (both volumes measured in $\mathrm{L}$ ), and $\Delta C_{\mathrm{w}} \mathrm{O}_{2} / \Delta t$ is the rate at which the oxygen concentration decreased over time $\left(\mathrm{mg} \mathrm{O}_{2} \mathrm{~L}^{-1}\right.$ $\mathrm{h}^{-1}$ ), after adjusting for changes in temperature and barometric pressure.

Preparation of Muscle and Liver Homogenate for the Determination of Mitochondrial Function

Following their collection (see above), thin pieces of tissue (around $140 \mathrm{mg}$ of skeletal white muscle and $60 \mathrm{mg}$ of liver) were cut using microdissecting scissors to obtain a homogenous solution with particle size lower than $0.5 \mathrm{~mm}$ (tested by pipetting through a 1-mL tip) in $1 \mathrm{~mL}$ MiR05. The entire procedure was carried out at $4^{\circ} \mathrm{C}$ and was completed within 15 min of the fish being culled. The quality and permeability of our tissue preparations were compared with chemical and mechanical permeabilization of the muscle and 
liver, respectively (as done in Kuznetsov et al. 2002 for the liver and in Larsen et al. 2014 for the muscle). These pilot studies showed that the permeability of the tissues, oxidative capacities, and integrity of the external mitochondrial membrane were similar to if not better than the homogenization methods of preparation (data not shown here). The tissue preparation used here allowed a rapid and efficient liver and muscle preparation, with no loss of tissue, while maintaining the quality of the mitochondria.

\section{Mitochondrial Respiration}

Mitochondrial respiration was measured using two Oxygraph$2 \mathrm{k}$ high-resolution respirometers (Oroboros Instruments, Innsbruck, Austria) running in parallel, each with two independent chambers. These respirometers allow high-resolution measurement of slow oxygen fluxes, which dropped on occasion to $20 \mathrm{pmol} \mathrm{O}_{2} \mathrm{~s}^{-1} \mathrm{~mL}^{-1}$ in our conditions. The oxygen electrodes were calibrated by two points: air-saturated MiR05 and zero oxygen after sodium dithionite addition. Oxygen concentration (nmol $\mathrm{mL}^{-1}$ ) and oxygen flux were recorded using DatLab software (Oroboros Instruments). Oxygen flux was expressed as picomoles per second per milligram, negative time derivative of oxygen concentration, divided by tissue mass per volume and corrected for instrumental background oxygen flux arising from oxygen consumption of the oxygen sensor and backdiffusion into the chamber (Pesta and Gnaiger 2012). The total titration protocol was completed within $2 \mathrm{~h}$.

The protocol was as follows: first the tricarboxylic acid cycle was reconstituted by adding pyruvate $(5 \mathrm{mM})$ and malate $(0.5 \mathrm{mM})$ to support the electron entry to complex I and succinate $(10 \mathrm{mM})$ to support the electron entry to complex II (Gnaiger 2009). Oxidative phosphorylation capacity was reached by adding a saturating concentration of $\mathrm{ADP}\left(1 \mathrm{mM} \mathrm{ADP}\right.$ with $\left.6 \mu \mathrm{M} \mathrm{Mg}^{2+}\right)$. Cytochrome c (Cytc; $10 \mu \mathrm{M})$ was then added as a quality control, with a Cytc stimulation of respiration rate below 15\% indicating acceptable mitochondrial membrane integrity (Kuznetsov et al. 2002). Leak respiration, that is, the oxidation to compensate for the proton leak and in the absence of phosphorylation, was induced by adding oligomycin $(2 \mu \mathrm{g} \mathrm{mL})$, an inhibitor of ATP synthase. Addition of complex I inhibitor $(0.5 \mu \mathrm{M}$ rotenone $)$ and complex III inhibitor $(2.5 \mu \mathrm{M}$ antimycin $\mathrm{A})$ determined residual oxygen consumption, which was then subtracted from all other values. Finally, COX respiration was measured by adding ascorbate $(2 \mathrm{mM})$ and $\mathrm{N}, \mathrm{N}, \mathrm{N}^{\prime}, \mathrm{N}^{\prime}$-tetramethyl-p-phenylenediamine dihydrochloride $(0.5 \mathrm{mM})$.

To avoid limitation of oxygen diffusion at low oxygen concentrations occurring in permeabilized tissue (Gnaiger et al. 1998), the sequential protocol was run under hyperoxygenation (400$650 \mu \mathrm{M}$ for the muscle and $100-650 \mu \mathrm{M}$ for the liver, which were found not to be oxygen sensitive within these ranges). Pure oxygen gas was added to the respirometry chamber at the very beginning of the titration and before the measurement of COX activity.

\section{Integrity of the Mitochondria}

Statistical Analysis

Paired $t$-tests were used to compare mitochondrial phosphorylating respiration in the absence and then presence of Cytc, to evaluate the quality of the mitochondria preparation estimated as the integrity of the outer mitochondrial membrane.

Results

Addition of Cytc to the liver preparation led to a statistically significant but biologically small elevation in the respiration rate (mean increase of $6.71 \% \pm 0.53 \%$, paired $t=-13.63, n=$ $40, P<0.001)$. Adding Cytc to the skeletal muscle preparation led to an even smaller increase in the respiration rate $(1.60 \% \pm$ $0.54 \%$, paired $t=-2.73, n=40, P=0.009$ ), again indicating that the tissue preparation was good. For both tissues, respiration rates in the presence of ADP plus Cytc were used when describing phosphorylating respiration.

Table A1: Parameters (intercept and slope $\beta \pm \mathrm{SE}$ ) from regression analyses

\begin{tabular}{lcccrr}
\hline Dependent variable & Independent variable & Intercept \pm SE & $\beta \pm$ SE & $r^{2}$ & $P$ \\
\hline SMR & $M$ & $-.365 \pm .170$ & $.446 \pm .158$ & .182 & $\mathbf{. 0 0 8}$ \\
MMR & $M$ & $.026 \pm .090$ & $.782 \pm .083$ & .709 & $<.001$ \\
Liver LEAK & Liver COX & $.984 \pm .580$ & $.028 \pm .013$ & .116 &. $\mathbf{0 3 9}$ \\
Liver OXPHOS & Liver COX & $-4.675 \pm 2.624$ & $.525 \pm .059$ & .695 & $<.001$ \\
Total liver LEAK & Total liver COX & $42.159 \pm 40.090$ & $.043 \pm .007$ & .533 & $<.001$ \\
Total liver OXPHOS & Total liver COX & $-359.236 \pm 196.068$ & $.483 \pm .033$ & .859 & $<.001$ \\
Muscle LEAK & Muscle COX & $.606 \pm .227$ & $.014 \pm .010$ & .049 & .180 \\
Muscle OXPHOS & Muscle COX & $7.504 \pm .860$ & $.137 \pm .039$ & .259 & $\mathbf{. 0 0 1}$ \\
\hline
\end{tabular}

Note. Regression analyses of standard metabolic rate (SMR) and maximum metabolic rate (MMR) as a function of body mass ( $M$ ) and regression analyses of mass-specific mitochondrial leak respiration (LEAK) and mass-specific mitochondrial phosphorylative respiration (OXPHOS) as a function of mass-specific cytochrome c oxidase (COX) activity of the liver and white muscle of juvenile brown trout Salmo trutta. Total liver mitochondrial respiration was calculated as mass-specific mitochondrial respiration multiplied by the total wet mass of the liver. SMR, MMR, and $M$ were log transformed before the regression analyses. Bold denotes significance. 


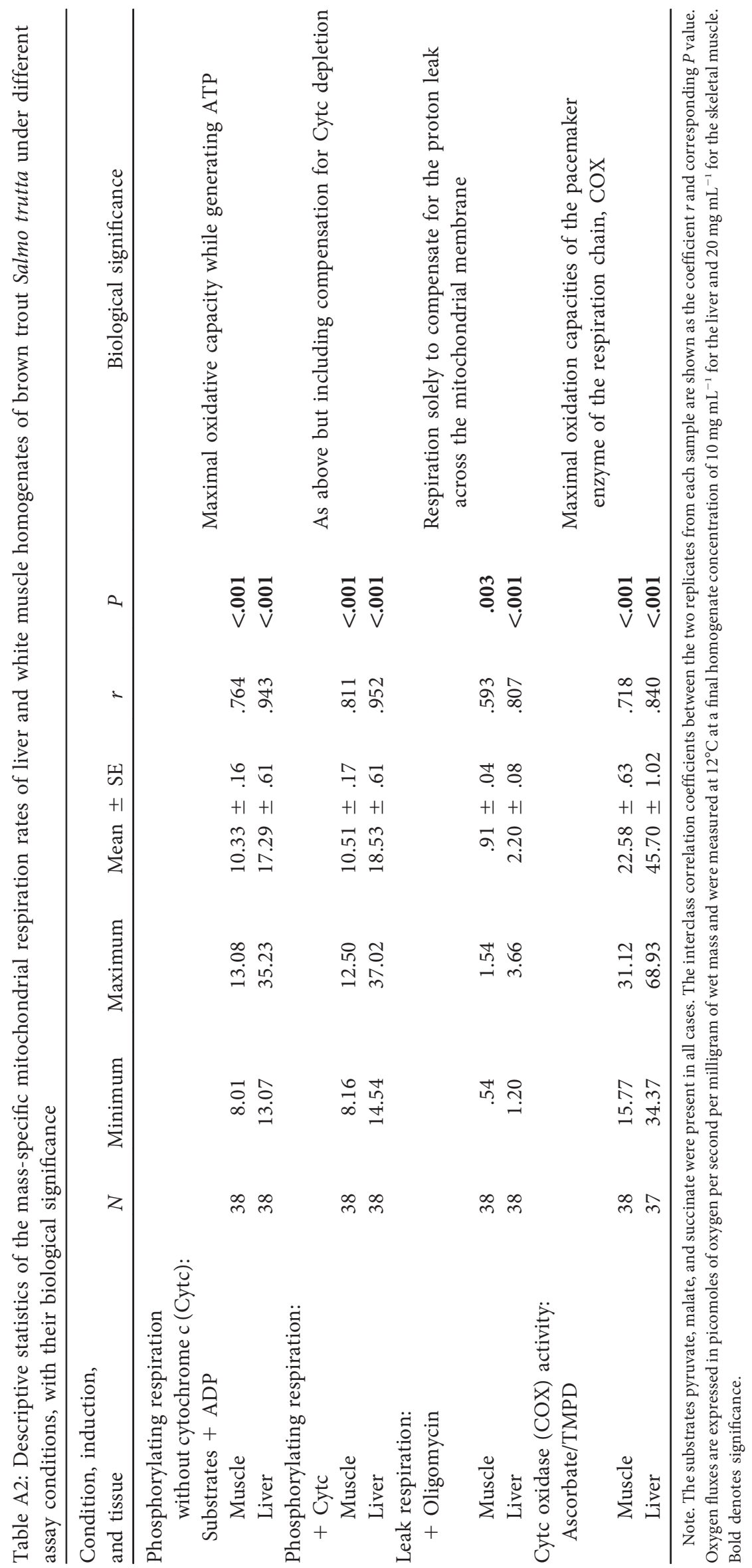

This content downloaded from 130.209.006.041 on October 03, 2016 05:50:46 AM 


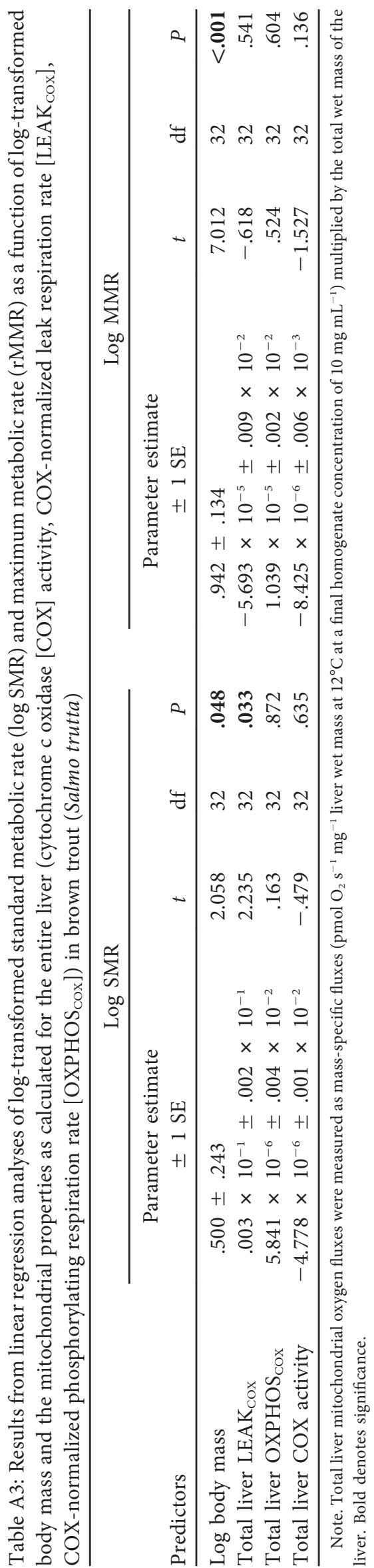

This content downloaded from 130.209.006.041 on October 03, 2016 05:50:46 AM 


\section{Literature Cited}

Allen J.L. 1988. Residues of benzocaine in rainbow trout, largemouth bass, and fish meal. Progress Fish-Cult 50:59-60.

Amara C.E., E.G. Shankland, S.A. Jubrias, D.J. Marcinek, M.J. Kushmerick, and K.E. Conley. 2007. Mild mitochondrial uncoupling impacts cellular aging in human muscles in vivo. Proc Natl Acad Sci USA 104:1057-1062.

Auer S.K., K. Salin, G.J. Anderson, and N.B. Metcalfe. $2015 a$. Aerobic scope explains individual variation in feeding capacity. Biol Lett 11:20150793.

Auer S.K., K. Salin, A.M. Rudolf, G.J. Anderson, and N.B. Metcalfe. 2015b. Flexibility in metabolic rate confers a growth advantage under changing food availability. L Anim Ecol 84:1405-1411.

. 2015c. The optimal combination of standard metabolic rate and aerobic scope for somatic growth depends on food availability. Funct Ecol 29:479-486.

Bennett A.F. and J.A. Ruben. 1979. Endothermy and activity in vertebrates. Science 206:649-654.

Biro P.A. and J.A. Stamps. 2010. Do consistent individual differences in metabolic rate promote consistent individual differences in behavior? Trends Ecol Evol 25:653-659.

Boldsen M.M., T. Norin, and H. Malte. 2013. Temporal repeatability of metabolic rate and the effect of organ mass and enzyme activity on metabolism in European eel $(A n-$ guilla anguilla). Comp Biochem Physiol A 165:22-29.

Brand M.D. and D.G. Nicholls. 2011. Assessing mitochondrial dysfunction in cells. Biochem I35:297-312.

Brookes P.S., J.A. Buckingham, A.M. Tenreiro, A.J. Hulbert, and M.D. Brand. 1998. The proton permeability of the inner membrane of liver mitochondria from ectothermic and endothermic vertebrates and from obese rats: correlations with standard metabolic rate and phospholipid fatty acid composition. Comp Biochem Physiol 119:325-334.

Brown J.C.L., D.J. Chung, K.R. Belgrave, and J.F. Staples. 2012. Mitochondrial metabolic suppression and reactive oxygen species production in liver and skeletal muscle of hibernating thirteen-lined ground squirrels. Am I Physiol 302:R15-R28.

Brown J.H., J.F. Gillooly, A.P. Allen, V.M. Savage, and G.B. West. 2004. Toward a metabolic theory of ecology. Ecology 85:1771-1789.

Burton T., S.S. Killen, J.D. Armstrong, and N.B. Metcalfe. 2011. What causes intraspecific variation in resting metabolic rate and what are its ecological consequences? Proc R Soc B 278: 3465-3473.

Careau V. and T. Garland Jr. 2012. Performance, personality, and energetics: correlation, causation, and mechanism. Physiol Biochem Zool 85:543-571.

Careau V., M.E. Gifford, and P.A. Biro. 2014a. Individual (co) variation in thermal reaction norms of standard and maximal metabolic rates in wild-caught slimy salamanders. Funct Ecol 28:1175-1186.

Careau V., B. Hoye, T. O'Dwyer, and B. Buttemer. $2014 b$. Among- and within-individual correlations between basal and maximal metabolic rates in birds. LExp Biol 217:3593-3596.
Chance B. and G. Williams. 1955. Respiratory enzymes in oxidative phosphorylation. III. The steady state. LBiol Chem 217:409-428.

Chappell M.A., T. Garland Jr., E.L. Rezende, and F.R. Gomes. 2004. Voluntary running in deer mice: speed, distance, energy costs and temperature effects. LExp Biol 207:3839-3854.

Chappell M.A., T. Garland Jr., G.F. Robertson, and W. Saltzman. 2007. Relationships among running performance, aerobic physiology and organ mass in male Mongolian gerbils. LExp Biol 210:4179-4197.

Chung D., G.P. Lloyd, R.H. Thomas, C.G. Guglielmo, and J.F. Staples. 2011. Mitochondrial respiration and succinate dehydrogenase are suppressed early during entrance into a hibernation bout, but membrane remodeling is only transient. L Comp Physiol B 181:699-711.

Clark T.D., E. Sandblom, and F. Jutfelt. 2013. Aerobic scope measurements of fishes in an era of climate change: respirometry, relevance and recommendations. LExp Biol 216: 2771-2782.

Coen P.M., S.A. Jubrias, G. Distefano, F. Amati, D.C. Mackey, N.W. Glynn, T.M. Manini, et al. 2012. Skeletal muscle mitochondrial energetics are associated with maximal aerobic capacity and walking speed in older adults. IGerontol A 68: 447-555.

Elliott J.M. 1976. The energetics of feeding, metabolism and growth of brown trout (Salmo trutta L.) in relation to body weight, water temperature and ration size. LAnim Ecol 45: 923-948.

Farrell A.P., S.G. Hinch, S.J. Cooke, D.A. Patterson, G.T. Crossin, M. Lapointe, and M.T. Mathes. 2008. Pacific salmon in hot water: applying aerobic scope models and biotelemetry to predict the success of spawning migrations. Physiol Biochem Zool 81:697-708.

Fry F.E.J. 1947. Effects of an environment on animal activity. Univ Tor Stud Biol Ser 55:1-62.

Garland T., Jr. 1984. Physiological correlates of locomotory performance in a lizard: an allometric approach. Am I Physiol 247:R806-R815.

Garland T., Jr., and P.L. Else. 1987. Seasonal, sexual, and individual variation in endurance and activity metabolism in lizards. Am I Physiol 252:R439-R449.

Gifford M.E., T.A. Clay, and V. Careau. 2014. Individual (co) variation in standard metabolic rate, feeding rate, and exploratory behavior in wild-caught semiaquatic salamanders. Physiol Biochem Zool 87:384-396.

Gnaiger E. 2009. Capacity of oxidative phosphorylation in human skeletal muscle: new perspectives of mitochondrial physiology. Int I Biochem Cell Biol 41:1837-1845.

Gnaiger E., B. Lassnig, A. Kuznetsov, G. Rieger, and R. Margreiter. 1998. Mitochondrial oxygen affinity, respiratory flux control and excess capacity of cytochrome c oxidase. LExp Biol 201:1129-1139.

Gomes F.R., J.G. Chaui-Berlinck, J. Bicudo, and C.A. Navas. 2004. Intraspecific relationships between resting and activity metabolism in anuran amphibians: influence of ecology and behavior. Physiol Biochem Zool 77:197-208. 
Guderley H. and H.O. Pörtner. 2010. Metabolic power budgeting and adaptive strategies in zoology: examples from scallops and fish. Can I Zool 88:753-763.

Harper J.A., K. Dickinson, and M.D. Brand. 2001. Mitochondrial uncoupling as a target for drug development for the treatment of obesity. Obes Rev 2:255-265.

Houlihan D.F., D.N. McMillan, and P. Laurent. 1986. Growth rates, protein synthesis, and protein degradation rates in rainbow trout: effects of body size. Physiol Zool 59:482-493.

Hulbert A.J. and P.L. Else. 2000. Mechanisms underlying the cost of living in animals. Annu Rev Physiol 62:207-235.

Hulbert A.J., N. Turner, J. Hinde, P. Else, and H. Guderley. 2006. How might you compare mitochondria from different tissues and different species? L Comp Physiol B 176:93-105.

Jacobs R.A., C. Siebenmann, M. Hug, M. Toigo, A.-K. Meinild, and C. Lundby. 2012. Twenty-eight days at 3454-m altitude diminishes respiratory capacity but enhances efficiency in human skeletal muscle mitochondria. FASEB I 26:5192-5200.

John-Alder H.B. 1983. Effects of thyroxine supplementation on metabolic rate and aerobic capacity in a lizard. Am Physiol 244:R659-R666.

Kadenbach B. 2003. Intrinsic and extrinsic uncoupling of oxidative phosphorylation. Biochim Biophys Acta 1604:77-94.

Khan J.R., F.I. Iftikar, N.A. Herbert, E. Gnaiger, and A.J.R. Hickey. 2014. Thermal plasticity of skeletal muscle mitochondrial activity and whole animal respiration in a common intertidal triplefin fish, Forsterygion lapillum (family: Tripterygiidae). I Comp Physiol B 184:991-1001.

Konarzewski M. and A. Ksiazek. 2013. Determinants of intraspecific variation in basal metabolic rate. $L$ Comp Physiol B 183:27-41.

Kuznetsov A.V., D. Strobl, E. Ruttmann, A. Konigsrainer, R. Margreiter, and E. Gnaiger. 2002. Evaluation of mitochondrial respiratory function in small biopsies of liver. Anal Biochem 305:186-194.

Kuznetsov A.V., V. Veksler, F.N. Gellerich, V. Saks, R. Margreiter, and W.S. Kunz. 2008. Analysis of mitochondrial function in situ in permeabilized muscle fibers, tissues and cells. Nat Protoc 3: 965-976.

Larsen F.J., T.A. Schiffer, K. Sahlin, B. Ekblom, E. Weitzberg, and J.O. Lundberg. 2011. Mitochondrial oxygen affinity predicts basal metabolic rate in humans. FASEB I 25:2843-2852.

Larsen S., R. Kraunsoe, M. Gram, E. Gnaiger, J.W. Helge, and F. Dela. 2014. The best approach: homogenization or manual permeabilization of human skeletal muscle fibers for respirometry? Anal Biochem 446:64-68.

Larsen S., J. Nielsen, C.N. Hansen, L.B. Nielsen, F. Wibrand, N. Stride, H.D. Schroder, et al. 2012. Biomarkers of mitochondrial content in skeletal muscle of healthy young human subjects. LPhvsiol (Lond) 590:3349-3360.

Mathot K.J. and N.J. Dingemanse. 2015. Energetics and behavior: unrequited needs and new directions. Trends Ecol Evol 30:199-206.

Monternier P.-A., V. Marmillot, J.-L. Rouanet, and D. Roussel. 2014. Mitochondrial phenotypic flexibility enhances energy savings during winter fast in king penguin chicks. LExp Biol 217:2691-2697.

Moyes C.D., P.M. Schulte, and P.W. Hochachka. 1992. Recovery metabolism of trout white muscle: role of mitochondria. Am I Physiol 262:R295-R304.

Norin T. and H. Malte. 2011. Repeatability of standard metabolic rate, active metabolic rate and aerobic scope in young brown trout during a period of moderate food availability. L Exp Biol 214:1668-1675.

. 2012. Intraspecific variation in aerobic metabolic rate of fish: relations with organ size and enzyme activity in brown trout. Physiol Biochem Zool 85:645-656.

Norin T., H. Malte, and T.D. Clark. 2014. Aerobic scope does not predict the performance of a tropical eurythermal fish at elevated temperatures. LExp Biol 217:244-251.

Park S.-Y., J.R. Gifford, R.H.I. Andtbacka, J.D. Trinity, J.R. Hyngstrom, R.S. Garten, N.A. Diakos, et al. 2014. Cardiac, skeletal, and smooth muscle mitochondrial respiration: are all mitochondria created equal? Am J Physiol 307:H346H352.

Pesta D. and E. Gnaiger. 2012. High-resolution respirometry: OXPHOS protocols for human cells and permeabilized fibers from small biopsies of human muscle. Pp. 25-58 in C.M. Palmeira and A.J. Moreno, eds. Mitochondrial bioenergetics: methods and protocols. Humana, Totowa.

Pickering A.D., T.G. Pottinger, and P. Christie. 1982. Recovery of the brown trout, Salmo trutta L., from acute handling stress: a time-course study. IFish Biol 20:229-244.

Porter R.K. and M.D. Brand. 1995. Causes of differences in respiration rate of hepatocytes from mammals of different body-mass. Am I Physiol 269:R1213-R1224.

Rolfe D.F.S. and M.D. Brand. 1996. Contribution of mitochondrial proton leak to skeletal muscle respiration and to standard metabolic rate. Am I Physiol 271:C1380-C1389.

- 1997. The physiological significance of mitochondrial proton leak in animal cells and tissues. Biosci Rep 17:9-16. Rolfe D.F.S. and G.C. Brown. 1997. Cellular energy utilization and molecular origin of standard metabolic rate in mammals. Physiol Rev 77:731-758.

Rosenfeld J., T. Van Leeuwen, J. Richards, and D. Allen. 2015. Relationship between growth and standard metabolic rate: measurement artefacts and implications for habitat use and life-history adaptation in salmonids. L Anim Ecol 84:4-20.

Salin K., S.K. Auer, G.J. Anderson, C. Selman, and N.B. Metcalfe. 2016. Inadequate food intake at high temperatures is related to depressed mitochondrial respiratory capacity. LExp Biol 219: 1356-1362.

Salin K., S.K. Auer, B. Rey, C. Selman, and N.B. Metcalfe. 2015a. Variation in the link between oxygen consumption and ATP production, and its relevance for animal performance. Proc R Soc B 282:20151028.

Salin K., S.K. Auer, A.M. Rudolf, G.J. Anderson, A.G. Cairns, W. Mullen, R.C. Hartley, C. Selman, and N.B. Metcalfe. $2015 b$. Individuals with higher metabolic rates have lower levels of reactive oxygen species in vivo. Biol Lett 11:20150538. 
Salin K., E. Luquet, B. Rey, D. Roussel, and Y. Voituron. 2012. Alteration of mitochondrial efficiency affects oxidative balance, development and growth in frog (Rana temporaria) tadpoles. L Exp Biol 215:863-869.

Sandblom E., A. Grans, M. Axelsson, and H. Seth. 2014. Temperature acclimation rate of aerobic scope and feeding metabolism in fishes: implications in a thermally extreme future. Proc R Soc B 281:9.

Schlagowski A.I., F. Singh, A.L. Charles, F. Piquard, B. Geny, and J. Zoll. 2013. Efficiency of skeletal muscle mitochondrial coupling is a critical factor for maximal exercise capacity and oxygen uptake in rats. Fundam Clin Pharmacol 27: 33.

Selman C., S. Lumsden, L. Bunger, W.G. Hill, and J.R. Speakman. 2001. Resting metabolic rate and morphology in mice (Mus musculus) selected for high and low food intake. [Exp Biol 204: 777-784.
Swanson D.L., N.E. Thomas, E.T. Liknes, and S.J. Cooper. 2012. Intraspecific correlations of basal and maximal metabolic rates in birds and the aerobic capacity model for the evolution of endothermy. PLoS ONE 7:e34271.

Trzcionka M., K.W. Withers, M. Klingenspor, and M. Jastroch. 2008. The effects of fasting and cold exposure on metabolic rate and mitochondrial proton leak in liver and skeletal muscle of an amphibian, the cane toad Bufo marinus. LExp Biol 211: 1911-1918.

Vindenes Y. and Ø. Langangen. 2015. Individual heterogeneity in life histories and eco-evolutionary dynamics. Ecol Lett 18 : 417-432.

Willmer P., G. Stone, and I. Johnston. 2005. Environmental physiology of animals. Blackwell, Oxford.

Wood C.M. 1991. Acid-base and ion balance, metabolism, and their interactions, after exhaustive exercise in fish. J Exp Biol 160:285-308. 